\title{
Disulfide bridge formation prevents CaMKII/Calmodulin interaction in Parkinson's disease
}

Roberto Di Maio ${ }^{a}$, Ignacio J. General ${ }^{b}$, Emily Furbee ${ }^{c}$, Joseph C. Ayoobc , Sandra L. Castro $^{a}$, Ivet Baharc ${ }^{c}$, J. Timothy Greenamyre ${ }^{\mathrm{a}}$ and Filippo Pullara ${ }^{\mathrm{d}^{*}}$

a. Pittsburgh Institute for Neurodegenerative Diseases, University of Pittsburgh, b. School of Science and Technology, Universidad Nacional de San Martin Argentina, c. Department of Computational and Systems Biology University of Pittsburgh, d. SpIntellx Inc.

* correspondence should be addressed to Filippo Pullara: filippo.pullara@gmail.com

\section{Abstract}

There is increasing evidence for disordered $\mathrm{Ca}^{2+}$ signaling in dopamine neurons in Parkinson's disease (PD), and this likely involves altered $\mathrm{Ca}^{2+} /$ calmodulin-dependent protein kinase II (CaMKII) function. Previous work suggests that oxidative stress - a major feature in PD pathogenesis - affects regulatory methionine residues that sustain CaMKII activity in a $\mathrm{Ca}^{2+} / \mathrm{CaM}$-independent manner. Here, applying computational modeling, we predicted formation of a defined disulfide bridge close to the CaMKII docking site for $\mathrm{Ca}^{2+} / \mathrm{CaM}$ binding. In vitro and in vivo investigations using PD models revealed formation of a disulfide bridge and loss of the CaMKII-calmodulin interaction. Mutagenesis of the relevant cysteine residues abrogated disulfide bridge formation and recovered the CaMKII-calmodulin interaction. Importantly, dopamine neurons from post-mortem PD brain specimens also lost this regulatory protein-protein interaction, providing relevance in the human disease. This study provides novel insights into oxidative CaMKII-CaM dysfunction, which may contribute to the pathophysiology of PD. 
$\mathrm{Ca}^{2+} /$ calmodulin dependent protein kinase II, CaMKII, is a serine/threonine specific kinase regulated by the $\mathrm{Ca}^{2+} /$ calmodulin $\left(\mathrm{Ca}^{2+} / \mathrm{CaM}\right)$ complex. CaMKII is involved in a large number of biological processes, including $\mathrm{Ca}^{2+}$ regulation [1], the cell cycle [2], and T-cell activation [3]. CaMKII also regulates neuronal processes, such as long-term potentiation (LTP) and the modulation of neuronal excitability.

There are 4 CaMKII genes in humans, $\alpha, \beta, y$ and $\delta$, which have high sequence identity and are expressed in at least 38 isoforms [4] through alternative splicing. In the brain, CaMKIl $\alpha$ performs its function as a dodecameric protein holocomplex. This complex is composed of two stacked rings of six subunits each. Each subunit of the system is composed of three subdomains (Figure 1): 1. the C-terminal hub, located towards the center of the ring; 2 . the $\mathrm{N}$-terminal kinase head; and, 3. a linker that connects the hub with the kinase head. In the dodecameric complex, when the hub and kinase head are in close proximity, the protein conformation is called "closed" or "auto-inhibited," because it is not bound to the $\mathrm{Ca}^{2+} / \mathrm{CaM}$ complex. Conversely, when the hub and kinase head are far apart, the linker is extended in the active "open" conformation, and CaMKIl $\alpha$ may bind the $\mathrm{Ca}^{2+} / \mathrm{CaM}$. The mechanism governing the transition between the autoinhibited and active conformations is modulated by the phosphorylation of several amino acids. However, the molecular mechanisms of this regulation are still not well understood.

Other conformational changes in CaMKIl $\alpha$ or modifications such as disulfide (S-S) bridge formation may play a role in this regulation. The latter is particularly intriguing 
given that both CaMKIl $\alpha$ dysfunction and oxidative stress, which can induce S-S bridges in proteins and protein complexes, are both implicated in neurodegenerative diseases. However, despite the large amount of information on both the physiopathology of CamKIl $\alpha$ in the brain and the ubiquitous presence of oxidative conditions accompanying neurodegeneration, the link between these two phenomena remains under-explored. Furthermore, there is no strong evidence of redox modulation of CaMKII activity/function in brain, though recent studies report redox sensitive properties of CaMKII in the heart and lung [5]. One reason for this is that currently there are no available experimental structures of CaMKIl $\alpha$ protein dodecameric complexes with subunits in the open and active conformation. The only structure available of CaMKII in an open conformation is a crystal structure of a CaMKII $\delta$ monomer (PDB-ID 2WEL). The most complete CaMKII structure appeared in 2011 [6] and describes the structure of a CaMKIl $\alpha$ splice variant with all subunits in the closed/inactive conformation. A more complete structural analysis of CaMKII in various conformers and under different conditions, particularly redox modulation, would be an important next step in providing a more detailed mechanism of its regulation and deciphering the role of CaMKII in neurodegenerative diseases.

Dysregulation of $\mathrm{Ca} 2+$ homeostasis and signaling, and redox imbalance, have been suggested as key factors in various forms of neurodegeneration, including PD [7]. While most neuronal types use $\mathrm{Na}^{+}$to generate action potentials, nigrostriatal dopamine (DA) neuronal activity is characterized by spontaneous firing that relies on $\mathrm{Ca}^{2+}$ currents 
mediated by CaV1.3 channels. In at least some neuronal populations, CaV1.3 forms a complex with CaMKII that modulates channel function [8].

The $\mathrm{Ca}^{2+}$-specific physiology of substantia nigra dopamine neurons requires precise regulation of certain systems, including CAMKII-CaM-mediated signaling and mitochondrial $\mathrm{Ca}^{2+}$ uptake to avoid elevated and harmful concentrations of cytosolic $\mathrm{Ca}^{2+}$. Such $\mathrm{Ca}^{2+}$ cycling and signaling is associated with ROS production. In addition, excess ROS may derive directly from DA metabolism, which generates harmful byproducts, such as semiquinones and hydrogen peroxide [9]. The overall result is an increase in the basal levels of ROS in nigrostriatal neurons that reduces their ability to tolerate further oxidative insults. In this context, systemic administration of rotenone or paraquat, which causes ROS production, and which targets every cell in central nervous system, results in selective damage of the DA system, and, reproduces aspects of PD pathogenesis $[10,11]$.

In this study, we undertook a computational modeling approach to probe the dynamics and regulation of a CaMKII dodecameric complex. Here we provide evidence of oxidatively-induced formation of a functional S-S bridge in the docking site of CaMKII for $\mathrm{Ca}^{2+} / \mathrm{CaM}$ complex, which interferes with the normal CaMKII- $\mathrm{Ca}^{2+} / \mathrm{CaM}$ interaction. Computational approaches and mutagenesis experiments confirmed that residues C30 and C289, belonging to two distinct and adjacent CaMKII subunits, form this bridge. Moreover, we provide evidence of an oxidatively-mediated, drastic reduction of the 
CaMKII- $\mathrm{Ca}^{2+} / \mathrm{CaM}$ interaction in human idiopathic $\mathrm{PD}$ and in the rotenone rat model thereof, suggesting that this phenomenon may be relevant in PD pathogenesis.

\section{Results}

Examining the structural dynamics of CaMKIl $\alpha$ required a structure with protein subunits in the open/active conformation within the dodecameric complex. In the absence of such a structure, we used existing crystal structures of CaMKIl $\alpha$ in the closed conformation and CaMKII $\delta$ in an open configuration to create a model of a CaMKIll $\alpha$ dodecamer with four open subunits and eight closed subunits (see Methods). We ran 8 independent all atoms Molecular Dynamics simulations with explicit water (see Methods) of the modeled dodecamer, for a total simulation time of around $2.6 \mu \mathrm{s}$. We evaluated the distance between the $\alpha$-carbons of all of the cysteines and identified two cysteines, $\mathrm{C} 30$ and $\mathrm{C} 289$, belonging to adjacent subunits that get very close to each other due to the overall motions of the protein complex (Figure $1 \mathrm{~B}-\mathrm{C}$ ).

The analysis of the $\mathrm{C} \alpha-\mathrm{C} \alpha$ distances of $\mathrm{C} 30$ and $\mathrm{C} 289$ in independent simulations shows different outcomes emphasizing the stochasticity of the phenomenon. We found that in 4 cases the close proximity between Cas $(<10 \AA)$ is transient and it survives for just few ns. In two of the simulations we ran, the C30-C289 interaction is rare or does not occur. Finally, in the remaining 2 cases, the distance between those two cysteines stabilizes around $10-15 \AA$ for a significant period of time (Figure 1 B-C). 
In most of the simulations, after around 100ns, the $\mathrm{C} \alpha-\mathrm{C} \alpha$ distance drops to less than $20 \AA \AA$ with a minimum separation below $6 \AA$. We observe in our modeling that C30-C289 remain generally in close proximity. This suggests that in oxidative stress conditions, which is a hallmark of neurodegenerative diseases, it is possible that the interaction between the thiol groups of C30 and C289 will lead to a S-S bridge formation. Moreover, when C30 and C289 are in close proximity, they occlude the region where CaM binds (Threonine residues 305 and 306) [12, 13], which is otherwise accessible (Figure 2). Additionally, the Threonine residue, T286, whose phosphorylation is known to be one of the first steps in CaMKII activation [14-16], would similarly be strongly perturbed if a S-S bridge were to be formed between C30 and C289. These computational results suggest the presence of a S-S bridge between adjacent CaMKII subunits in the CaM biding region, which apparently would disrupt CaMKII-Ca ${ }^{2+} / \mathrm{CaM}$ binding, as well as CaMKII function.

To support our computational findings of a putative S-S bridge between adjacent subunits of CaMKII, we examined whether such a bond is formed under oxidative conditions in ventral midbrain primary neurons. We performed "redox western blot" assays, which allow detection of thiols derived from S-S bridges via an alkylating reaction with a $10 \mathrm{kDa}$ PEG-NEM tag, resulting in an observable $20 \mathrm{kDa}$ shift of the predicted molecular weight for each S-S bridge. In the absence of an oxidizing treatment, no significant shift of CaMKII is observed, whereas in cells exposed to the mitochondrial complex I inhibitor, rotenone, a $20 \mathrm{kDa}$ shift was observed for CaMKII 
(Figure $3 \mathrm{~A}-\mathrm{B}$ ), suggesting the formation of a single S-S bridge in CaMKII under oxidative stress conditions.

To identify which cysteine residues are involved in this S-S bridge, we set out to directly test our computational results that implicate C30 and C289 as the bridge-forming pair. We performed mutagenesis on human CaMKII $\alpha$ and expressed these mutant transgenes in a Drosophila S2R+ adherent cell line, to avoid cross-immunoreactivity with native proteins. Redox western blot assay in S2R+ wild type, C30A, C289A, and C30A:C289A mutants revealed that exposure to $5 \mathrm{nM} \mathrm{H}_{2} \mathrm{O}_{2}$ elicited formation of an S-S bridge (20 kDa MW shift in wild type CaMKIla), similar to that seen in the ventral midbrain neurons (Figure 3C-D). There was no MW shift observed under basal conditions. Likewise, no MW shifts were observed with either mutant alone, or in combination, under normal or oxidative stress conditions, adding strong support for a specific C30/C289-related S-S bridge in CaMKII $\alpha$.

In addition to revealing an S-S bridge in CaMKIl $\alpha$, our computational studies also indicate that $\mathrm{Ca}^{2+} \mathrm{CaM}$ binding to CaMKIl $\alpha$ is likely to be blocked when the $\mathrm{S}-\mathrm{S}$ bridge is formed between C30 and C289. To test this, we employed a proximity ligation assay, which provides fluorescent signal where there is interaction between two tagged proteins, in this case CaMKIl $\alpha$ and CaM. In rat ventral midbrain neurons, under basal conditions, we observed a strong CaMKIl $\alpha$ :CaM interaction (Figure 4 A-B). Under oxidative stress conditions induced by rotenone, the interaction between CaMKIl $\alpha$ and CaM was significantly reduced. We repeated these experiments in the Drosophila S2R+ 
cell line using C30A and C289A mutations, alone and in combination, to test the potential role of these cysteine residues in the CaMKIl $\alpha$ :CaM interaction. In both normal and oxidative stress conditions, the CaMKIl $\alpha$ :CaM interaction was only disrupted when the wild type protein was expressed in $\mathrm{H}_{2} \mathrm{O}_{2}$-treated cells (Figure 4 bottom-D). These results demonstrate that normal CaM binding to CaMKIl $\alpha$ is disrupted when the S-S bridge is formed between C30 and C289 when cells are under oxidative stress.

The mitochondrial complex I inhibitor, rotenone, reproduces many features of PDrelated pathogenic events including mitochondrial dysfunction, oxidative stress, altered Ca2+ homeostasis, and accumulation of toxic forms of a-synuclein [17-20]. We further assayed the CamKII $\alpha: \mathrm{Ca}^{2+} \mathrm{CaM}$ interaction in this paradigm to examine if the disruption of binding is manifested in the in vivo rat $\mathrm{PD}$ model. A robust CamKIl $\alpha: \mathrm{Ca}^{2+} \mathrm{CaM}$ interaction was observed in nigrostriatal neurons under basal conditions, but, we observed a significant reduction of CamKIl $\alpha: \mathrm{Ca}^{2+} \mathrm{CaM}$ interaction (by over $70 \%$ ) in dopamine neurons of substantia nigra pars compacta (SNpc) from rotenone-treated animals, which were exhibiting PD-like behaviors (Figure 5). Our results from rotenonetreated rats demonstrated an impaired CaMKII/CaM interaction. Moreover, previous evidence in PD models report CaMKII hyperactivity related to high levels of phosphorylation at the Thr 286 residue in in parallel with decreased activity of the specific phosphatase PP1Y1 [21, 22]. The event may be linked to an uncontrolled Calmodulin-independent CaMKII activity. Similarly, as (QQQ Add supplementary FIGUREfig S...) shows, we detected a significant increase of pThr286 in rotenone treated animals, suggesting that, in absence of CaM modulation, CaMKII exerts an 
uncontrolled Kinase activity. To determine whether this process is relevant to idiopathic human PD, we performed PL assays (CaMKII:CaM) in blinded postmortem substantia nigra sections from PD patients $(\mathrm{N}=5)$ and from controls $(\mathrm{N}=4)$. Compared to controls, nigrostriatal dopamine neurons from all PD cases showed very low PL signal, indicating loss of interaction between CaMKII and CaM (Figure 6). This evidence strongly suggests that CaM-mediated modulation of CaMKII is impaired in the human disease and may be a relevant factor in PD pathophysiology. Together, these results provide a strong case for an oxidative stress-induced S-S bridge in CaMKII that occludes calmodulin binding and contributes to the pathogenesis of PD. 


\section{Discussion}

In this study, we applied novel computational techniques in conjunction with experimental in vitro and in vivo approaches to provide new insights into the mechanisms of regulation of CaMKIl $\alpha$ and its interaction with calmodulin. Our computational results identified the presence of a putative S-S bridge across two adjacent CaMKII $\alpha$ subunits in its dodecameric configuration. Interestingly, this predicted bridged conformation occludes the binding site for calmodulin as well as the activation site for CaMKIl $\alpha$. In cell culture models, including ventral midbrain primary cultures, we confirmed the presence of this S-S bridge, which only occurs under oxidative stress conditions, and identified the specific cysteines involved. Further investigation demonstrated that formation of this bridge does in fact block CaMKII interactions with calmodulin, and again this only occurs in oxidative stress conditions. Mutating one or both cysteine residues that form the bridge both blocks S-S formation and restores calmodulin binding. In this context, we observed a significant loss of interaction CaMKIla/calmodulin in substantia nigra dopamine neurons of rotenone-treated animals and, most importantly, in dopamine neurons in post-mortem PD brain specimens. Our results, obtained with complementary experimental approaches, demonstrated for the first time a specifically-defined S-S bridge in CaMKIl $\alpha$ that affects proper modulatory control of CaMKII exerted by $\mathrm{Ca}^{2+} /$ calmodulin complex. This novel mechanism of CaMKIl $\alpha$ regulation under oxidative stress conditions, provides insights into the potential relevance of CaMKIl $\alpha$ dysfunction that may trigger a downstream pathological cascade of events culminating in PD-related neurodegeneration. Further studies are 
required to better define the role of oxidative CaMKII dysfunction in the pathophysiology of PD.

\section{Methods}

\section{Molecular Dynamics Computer Simulation}

Computational simulations of the CaMKIl $\alpha$ were performed using the AMBER15 [23] software package, with all of them-except for the minimizations—using the GPU version of the PMEMD program. The crystal structure of CaMKIl $\alpha$ in a closed/autoinhibited conformation (PDB structure 3SOA) was used as the starting structure [6] of human CaMKII in its $\alpha$ isoform, with a $\beta 7$ linker (the shortest linker in all known splice variants [24]. Some of the subunits in this dodecamer were converted computationally to open conformations based on the 2WEL PDB crystal structure of the CaMKIII isoform, which is in the open conformation. This was feasible since the kinase domains of both isoforms have a high sequence similarity of about $93 \%$. The final structure contained eight closed and four open sub-units of CaMKIl $\alpha$ in a dodecameric configuration (Figure 1).

In order to bring the modeled molecule to a stable conformation, we ran several cycles of minimization and equilibration. We employed the Amber12 force field and the TIP3P water model. The systems were kept at a temperature of $298 \mathrm{~K}$, using Langevin dynamics with a collision frequency of $2 \mathrm{ps}^{-1}$, and part of the protocol used pressure control, via a weak-coupling Berendsen barostat, with a relaxation time of 2 ps. The SHAKE algorithm was adopted, allowing the use of a 2 fs time step. The protocol followed for minimization and equilibration was: 1) 100 cycles of minimization, using the XMIN method, followed by 5,000 cycles using steepest descent and another 5,000 steps using conjugate gradient; 2) 1 ns of heating to $298 \mathrm{~K}$, followed 
by another ns at constant $\mathrm{T}$ and $\mathrm{P}$ ( $1 \mathrm{~atm})$, and finally $20 \mathrm{~ns}$ with constant $\mathrm{T}$. The simulation box consisted of one CaMKIl $\alpha$ dodecamer complex with eight subunits in the closed conformation and four subunits in the open conformation. All atom simulations were performed on CaMKIl $\alpha$ in a box with explicit water plus ions to neutralize electrostatic charges. Systems were $>1.3 \times 10^{6}$ atoms and all runs were $>300 n s$. For all the runs we used Nvidia GeForce TITAN-X GPUs.

\section{Experimental}

\section{Ventral midbrain primary cultures}

Neuronal cultures were prepared from embryonic day 17 Sprague-Dawley rats (Charles River, Wilmington, MA, USA). Embryos were obtained from two to three pregnant dams. Pooled ventral midbrain tissue was dissociated with enzymatic digestion using trypsin followed by mechanical trituration. Cells were seeded at a density of $5 \times 10^{5} /$ well in a 24 -well plate. The cultures were maintained at $37^{\circ} \mathrm{C}$ in a humidified atmosphere of $5 \% \mathrm{CO}_{2}$ and $95 \%$ air in MEM with 2\% FBS, 2\% HS, $1 \mathrm{~g} / \mathrm{L}$ glucose, $2 \mathrm{mM}$ GlutaMax, 100 IM non-essential amino acids, $1 \mathrm{mM}$ sodium pyruvate, $50 \mathrm{U} / \mathrm{mL}$ penicillin, and $50 \mathrm{lg} / \mathrm{mL}$ streptomycin. After $48 \mathrm{~h}$, the culture medium was replaced with serum-free Neurobasal medium containing $0.5 \mathrm{mg} / \mathrm{mL}$ AlbuMAX I, $2 \mathrm{mM}$ GlutaMAX I, 2\% B27 supplement, $50 \mathrm{U} / \mathrm{mL}$ penicillin, and $50 \mathrm{lg} / \mathrm{mL}$ streptomycin. $50 \mathrm{ng} / \mathrm{mL}$ GDNF (\#512-GF-050; R\&D Systems. Minneapolis, MN, USA) was also added. Five days after seeding, the media was removed and replenished with Neurobasal medium. At 9 days in vitro (DIV), fresh Neurobasal medium was added over old medium in combination with GDNF. At 13 DIV, $70 \%$ of old medium was replaced. All the experiments were performed at DIV 15 exposing cells to Vehicle (DMSO) or 50nM rptenone for 24 hours. All procedures were performed with the approval of the University of Pittsburgh Animal Care and Use Committee.

\section{Animals}


Six- to seven-month-old adult male Lewis rats were used for all experiments (Envigo). The animals were maintained under standard conditions of 12 hours light/dark cycle in a $22 \pm 1^{\circ} \mathrm{C}$ temperature-controlled room with 50\%-70\% humidity. Water and food were provided ad libitum. Animals were adapted for two weeks to the described conditions before the experiments. All studies were approved by the Institutional Animal Care and Use Committee at the University of Pittsburgh and were performed in accordance with published National Institutes of Health guidelines.

\section{Mutagenesis experiments}

CaMKIl $\alpha$ and calmodulin expression in Drosophila cells was accomplished as follows: CaMKIl $\alpha$ and calmodulin coding sequences were copied from human cDNA clones CAMK2A transcript variant 2 (catalog SC109000, Origene, Rockville MD) and CALM1 Human calmodulin 1 transcript variant 1 (catalog SC115829, Origene, Rockville MD), using PCR primers to add flanking restriction sites, with the following primers: EcoRI-CAMK2A-FWD; GAGAGAATTCATGGCCACCATCACCTGCAC, CAMK2A-STP-Sall-REV; TCTCGTCGACTTAGTGGGGCAGGACGGAGGGCG, Mfel-CALM1-FWD; GAGACAATTGATGGCTGATCAGCTGACCGA, CALM1-STP-Sall-REV; TCTCGTCGACTCATTTTGCAGTCATCATCT. Products were ligated into appropriately gapped vector pRmHA3 (Drosophila Genomics Research Center, Catalog 1145) to generate pRmHA3CaMKII and pRmHA3-CALM1. Point mutation variants (pRmHA3-CaMKII ${ }^{\mathrm{c} 30}$, pRmHA3$\mathrm{CaMKII}^{\mathrm{C289A}}$, and pRmHA3-CaMKII ${ }^{\mathrm{C30A}, \mathrm{C} 289 \mathrm{~A}}$ ) were generated by site directed mutagenesis of pRmHA3-CaMKII using QuickChange Lighting Kit (Agilent, Catalog 210518.) The full coding sequence of all pRmHA3-CaMKII constructs was verified by Sanger sequencing. pRmHA3CaMKII constructs were introduced to Drosophila S2 cells (Drosophila Genomics Research Center, catalog 181) cultured under standard conditions in Schneider's media with 10\% FBS and Pen-Strep at densities between $0.5-2.0 \times 10^{6}$, by transient Effectene transfection (Qiagen 
301425) according to manufacturer recommendations. Expression was induced with 500 uM CuSO4 at the time of transfection and transfected cells were incubated for 48 hours under standard conditions. pRmHA3-mCherry was co-transfected with pRmHA3-CaMKII constructs to evaluate transfection efficiency and rule out morphological indications of toxicity in transfected cells. Oxidative stress was induced by treating $\mathrm{S} 2$ cells with $0.5 \mathrm{mM} \mathrm{H}_{2} \mathrm{O}_{2}$ in normal culture media for thirty minutes before lysis for immunoblots as described below.

\section{In vitro and in vivo rotenone models of PD}

Primary cultures were exposed to $50 \mathrm{nM}$ rotenone (Sigma-Aldrich St. Louis, MO, USA) or vehicle (2\% DMSO) for 24 hours. At the endpoint, cells were fixed in $4 \%$ paraformaldehyde. Cells were incubated overnight with primary antibodies for CaMKII, calmodulin, and tyrosine hydroxylase, as marker of dopaminergic neurons.

For in vivo studies, animals were randomly divided into vehicle (6 animals) and rotenone (11 animals) groups of treatment. Before injections, body weights were recorded for each animal. Rotenone was administered intraperitoneally once a day at a dose of $2.8 \mathrm{mg} / \mathrm{kg}$ until the end of the treatment (11-13 days). The solution was prepared as a 50X stock dissolved in pure dimethyl sulfoxide at final concentration of 2\%, then diluted in Miglyol 812N (Sasol North America, Inc, Houston, TX; distributed by Warner Graham, Baltimore, MD, USA) at final concentration of $98 \%$, and administered at $1 \mathrm{~mL} / \mathrm{kg}$. This regimen produces relatively uniform bilateral nigrostriatal lesions, leading to loss of about $50 \%$ of dopaminergic neurons [20]. Control animals received an equivalent amount of vehicle ( $2 \%$ dimethyl sulfoxide $98 \%$ Miglyol). Tissues were collected from each animal when they developed the debilitating behavioral phenotypes of akinesia, rigidity, and postural instability.

\section{Histology}


Animals were euthanized by $\mathrm{CO}_{2}$ inhalation followed by decapitation. The brains were removed following an intracardial perfusion with saline solution ( $\mathrm{NaCl} 0.9 \%)$ and a fixing perfusion with $4 \%$ paraformaldehyde (PFA). After 48 hours, the brains were placed in $30 \%$ sucrose in phosphate buffered saline (PBS) for cryoprotection until infiltration was complete (at least 3 days).

\section{Proximity Ligation Assay}

Proximity Ligation Assay (PLA) (Duolink; Sigma Aldrich) was performed as described in https://reedd.people.uic.edu/ReedLabPLA.pdf in 4\% PFA-fixed tissue or cell cultures to assess the level of interaction CaMKII-Ca ${ }^{2+} / \mathrm{CaM}$ under our experimental conditions. Samples were incubated with specific primary antibodies against CaMKIl $\alpha$ (Rb anti- CaMKIl $\alpha$; ab92332 abcam) and calmodulin (Ms anti-calmodulin; ab2860 - abcam). PLA probes consisting of secondary antibodies (anti-Rb and anti-Ms) conjugated with complementary oligos (plus and minus respectively) were added to the reaction and incubated. Ligation solution, consisting of two oligos and ligase, was added. In this assay, the oligos hybridize to the two PLA probes and join to a closed loop if they are in close proximity. An amplification solution, consisting of nucleotides and fluorescently labeled oligos, was added together with polymerase. The oligonucleotide arm of one of the PLA probes acts as a primer for "rolling-circle amplification" (RCA) using the ligated circle as a template, and this generates a concatemeric product. Fluorescently labeled oligonucleotides hybridize to the RCA product. The PL signal is visible as a distinct fluorescent spot. Fluorescent images were captured using a confocal microscope (Olympus BX61, Fluoview 1000; Melville, NY, USA) and quantification was carried out at 60100X magnification. Control experiments included routine immunofluorescence staining of the proteins of interest under identical experimental conditions. 


\section{Redox Western Blot}

Levels of oxidized thiols (S-S) in CaMKII were measured with a redox Western blot technique. Ventral midbrain primary cultures or Drosophila cells were lysed in $50 \mathrm{mM}$ Tris- $\mathrm{HCl} \mathrm{pH} 7.0,2 \%$ sodium dodecyl sulfate (SDS), 1mM EDTA, protease inhibitor cocktail and $100 \mu \mathrm{M}$ N-ethylmaleimide (NEM), to stably alkylate free thiol residues $(\mathrm{SH})$. The lysate was heated at $65^{\circ} \mathrm{C}$ for $5 \mathrm{~min}$ to denature proteins and incubated for $20 \mathrm{~min}$ at room temperature. Proteins were precipitated in ice-cold acetone to remove un-reacted reagents, resuspended in 50mM Tris- $\mathrm{HCl}$ $\mathrm{pH} 7,2 \%$ SDS, and $10 \mathrm{mM}$ TCEP and heated at $65^{\circ} \mathrm{C}$ for $5 \mathrm{~min}$ and incubated 20 min at room temperature to reduce S-S bridges to free $\mathrm{SH}$ groups. Proteins were precipitated again, and the pellet was re-suspended in 50mMTris- $\mathrm{HCl} \mathrm{pH} \mathrm{7,2 \%} \mathrm{SDS,} \mathrm{and} 100 \mu \mathrm{M}$ polyethylene glycolmaleimide (PEG-NEM; 10KDa) to label the formerly oxidized thiols. After 20 min of incubation, the proteins were precipitated and a Western blot for CaMKIl $\alpha$ was performed. PEG-NEM labeling of formerly oxidized thiols increases the molecular weight of the protein by 10KDa for each labeled thiol. Blotted membranes were imaged with an Odyssey infrared scanner (LiCor), and the signal was quantified with the scanner's software.

\section{Statistical analyses}

Each result presented here was derived from three to six independent experiments. For simple comparisons of two experimental conditions, two-tailed, unpaired t-tests were used. Where variances were not equal, Welch's correction was used. For comparisons of multiple experimental conditions, one-way or two-way ANOVA was used, and if significant overall, post hoc corrections (Bonferroni or Sidak) for multiple pairwise comparisons were made. P-values less than 0.05 were considered significant. 


\section{Author contributions}

F.P. conceived the study, designed and carried out most of computer simulations and analysis. F.P. and R.D.M. designed the in vitro and in vivo experiments with input from J.T.G. and performed the data analysis. E.F and J.C.A. performed transfections of CaMKII mutants in fly cells. I.J.G. carried coarse grained analysis with input from I.B. and started the molecular dynamics simulations. S.L.C. performed all the histological assays in rat and human tissue. F.P., R.D.M., I.B., J.T.G. and J.C.A. contributed to the drafting of the manuscript. All authors discussed the results, commented and approved the manuscript.

\section{References}

1. Anderson, M.E., Calmodulin kinase signaling in heart: an intriguing candidate target for therapy of myocardial dysfunction and arrhythmias. Pharmacol Ther, 2005. 106(1): p. 39-55.

2. Skelding, K.A., J.A. Rostas, and N.M. Verrills, Controlling the cell cycle: the role of calcium/calmodulin-stimulated protein kinases I and II. Cell Cycle, 2011. 10(4): p. 631-9.

3. Lin, M.Y., et al., A pivotal role for the multifunctional calcium/calmodulindependent protein kinase II in T cells: from activation to unresponsiveness. J Immunol, 2005. 174(9): p. 5583-92.

4. Tombes, R.M., M.O. Faison, and J.M. Turbeville, Organization and evolution of multifunctional $\mathrm{Ca}(2+) / C a M-d e p e n d e n t$ protein kinase genes. Gene, 2003. 322: p. 17-31.

5. Erickson, J.R., et al., A dynamic pathway for calcium-independent activation of CaMKIl by methionine oxidation. Cell, 2008. 133(3): p. 462-74.

6. Chao, L.H., et al., A mechanism for tunable autoinhibition in the structure of a human Ca2+/calmodulin- dependent kinase II holoenzyme. Cell, 2011. 146(5): p. 732-45. 
7. Shioda, N. and K. Fukunaga, Physiological and Pathological Roles of CaMKIIPP1 Signaling in the Brain. Int J Mol Sci, 2017. 19(1).

8. Sahu, G., et al., Activity-Dependent Facilitation of CaV1.3 Calcium Channels Promotes KCa3.1 Activation in Hippocampal Neurons. J Neurosci, 2017. 37(46): p. 11255-11270.

9. Jenner, P. and C.W. Olanow, Oxidative stress and the pathogenesis of Parkinson's disease. Neurology, 1996. 47(6 Suppl 3): p. S161-70.

10. Betarbet, R., et al., Chronic systemic pesticide exposure reproduces features of Parkinson's disease. Nat Neurosci, 2000. 3(12): p. 1301-6.

11. McCormack, A.L., et al., Environmental risk factors and Parkinson's disease: selective degeneration of nigral dopaminergic neurons caused by the herbicide paraquat. Neurobiol Dis, 2002. 10(2): p. 119-27.

12. Hanson, P.I. and H. Schulman, Inhibitory autophosphorylation of multifunctional Ca2+/calmodulin-dependent protein kinase analyzed by site-directed mutagenesis. J Biol Chem, 1992. 267(24): p. 17216-24.

13. Colbran, R.J., Inactivation of Ca2+/calmodulin-dependent protein kinase II by basal autophosphorylation. J Biol Chem, 1993. 268(10): p. 7163-70.

14. Rellos, P., et al., Structure of the CaMKIIdelta/calmodulin complex reveals the molecular mechanism of CaMKII kinase activation. PLoS Biol, 2010. 8(7): p. e1000426.

15. Stratton, M., et al., Activation-triggered subunit exchange between CaMKII holoenzymes facilitates the spread of kinase activity. Elife, 2014. 3: p. e01610.

16. Pullara, F., E.K. Asciutto, and I.J. General, Mechanisms of Activation and Subunit Release in $\mathrm{Ca}(2+) / C a l m o d u l i n-D e p e n d e n t$ Protein Kinase II. J Phys Chem B, 2017. 121(45): p. 10344-10352.

17. Horowitz, M.P., et al., Single-cell redox imaging demonstrates a distinctive response of dopaminergic neurons to oxidative insults. Antioxid Redox Signal, 2011. 15(4): p. 855-71.

18. Sanders, L.H. and J. Timothy Greenamyre, Oxidative damage to macromolecules in human Parkinson disease and the rotenone model. Free Radic Biol Med, 2013. 62: p. 111-120.

19. Di Maio, R., et al., alpha-Synuclein binds to TOM20 and inhibits mitochondrial protein import in Parkinson's disease. Sci Transl Med, 2016. 8(342): p. 342ra78.

20. Greenamyre, J.T., et al., Lessons from the rotenone model of Parkinson's disease. Trends Pharmacol Sci, 2010. 31(4): p. 141-2; author reply 142-3.

21. Picconi, B., et al., Abnormal Ca2+-calmodulin-dependent protein kinase II function mediates synaptic and motor deficits in experimental parkinsonism. $\mathrm{J}$ Neurosci, 2004. 24(23): p. 5283-91.

22. Brown, A.M., et al., Association of protein phosphatase 1 gamma 1 with spinophilin suppresses phosphatase activity in a Parkinson disease model. J Biol Chem, 2008. 283(21): p. 14286-94.

23. D.A. Case, J.T. Berryman, R.M. Betz, D.S. Cerutti, T.E. Cheatham, III, T.A. Darden, R.E. Duke, T.J. Giese, H. Gohlke, A.W. Goetz, N. Homeyer, S. Izadi, P. Janowski, J. Kaus, A. Kovalenko, T.S. Lee, S. LeGrand, P. Li, T. Luchko, R. Luo, B. Madej, K.M. Merz, G. Monard, P. Needham, H. Nguyen, H.T. Nguyen, I. Omelyan, A. Onufriev, D.R. Roe, A. Roitberg, R. Salomon-Ferrer, C.L. 
Simmerling, W. Smith, J. Swails, R.C. Walker, J. Wang, R.M. Wolf, X. Wu, D.M. York and P.A. Kollman (2015), AMBER 2015, University of California, San Francisco

24. Wang, P., et al., Identification of alternative splicing variants of the beta subunit of human $\mathrm{Ca}(2+) /$ calmodulin-dependent protein kinase II with different activities. FEBS Lett, 2000. 475(2): p. 107-10. 

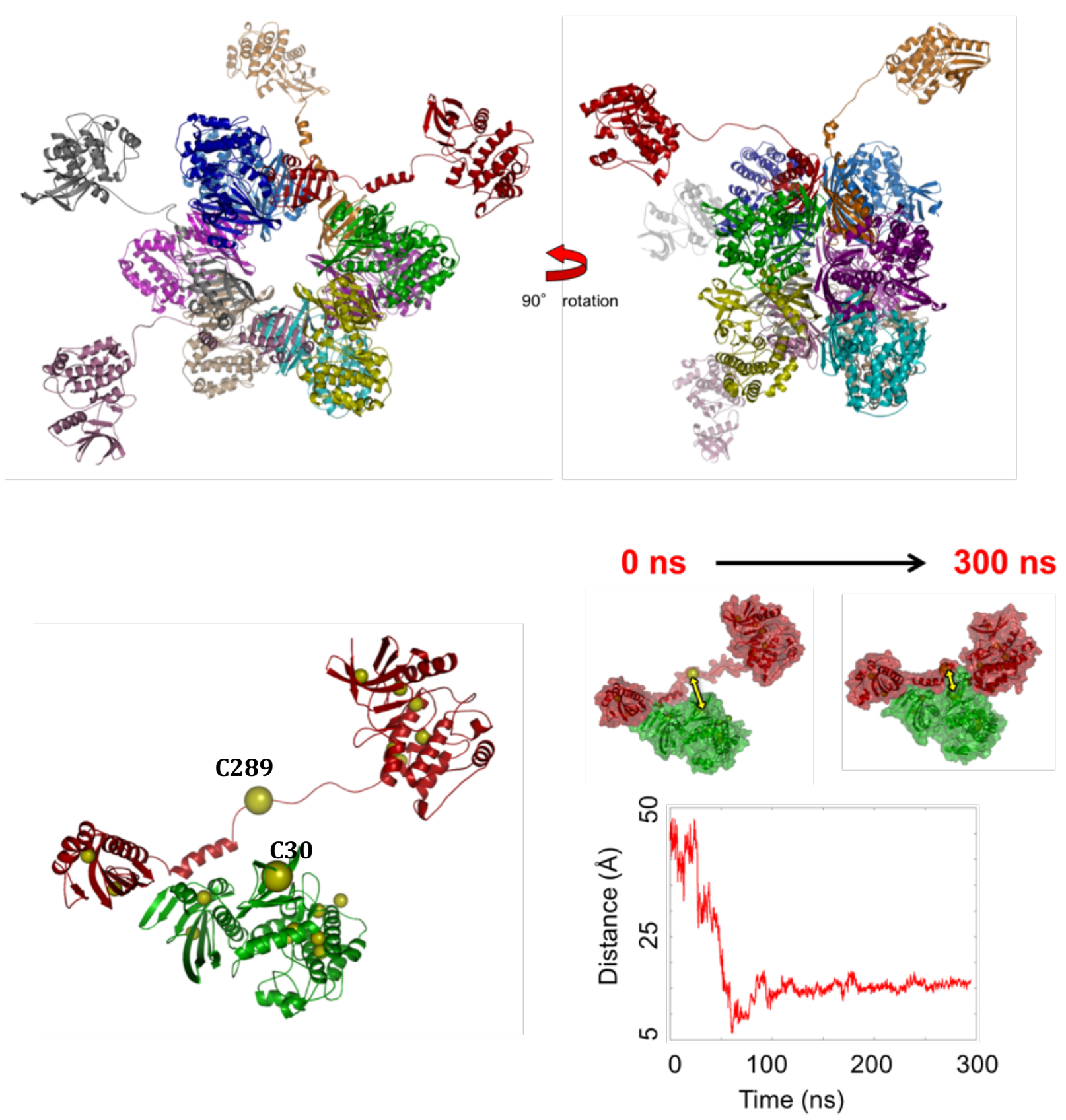

Figure 1

A: Top and side views of the full dodecamer model of CaMKIl $\alpha$. In this conformer there are four CaMKII subunits in the open conformation and the remaining eight in the closed conformation.

B: A detailed view of two adjacent CaMKII subunits. Represented as yellow spheres are the $\mathrm{C} \alpha$ of the cysteines. The $\mathrm{C} \alpha$ of $\mathrm{C} 30$ and $\mathrm{C} 289$ are represented by two larger yellow spheres. 
C: Snapshots from one of the all atoms molecular dynamics simulations highlighting the open and closed conformations. The double yellow arrows indicate the $\mathrm{C} \alpha-\mathrm{C} \alpha$ distance between $\mathrm{C} 30$ and $\mathrm{C} 289$. The graph shows the time evolution of the distance between those two residues. Note that the minimum $\mathrm{C} \alpha-\mathrm{C} \alpha$ distance reached during this simulation is less than $6 \AA$. 


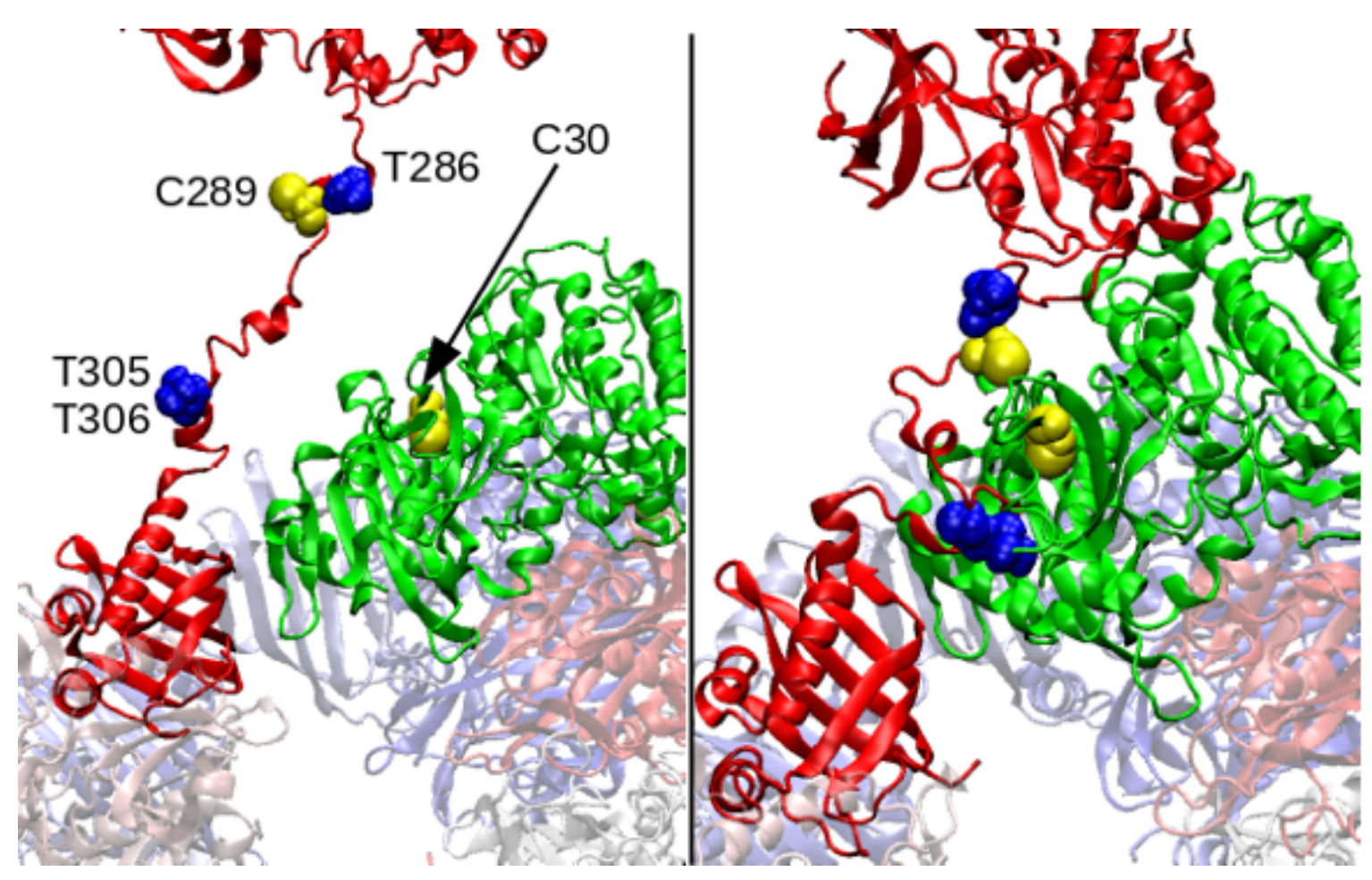

Figure 2

Close-up view of two subunits of CaMKII, showing the location of C30 and C289 (S-S bridge), T305 and T306 (binding region of CaM), and T286 (involved in CaMKII activation). When the bridge is not formed (left panel), there is room around T305/306 for CaM to bind. When the bridge is formed (right panel), the accessible area to the binding site gets greatly reduced, possibly preventing CaM binding. 


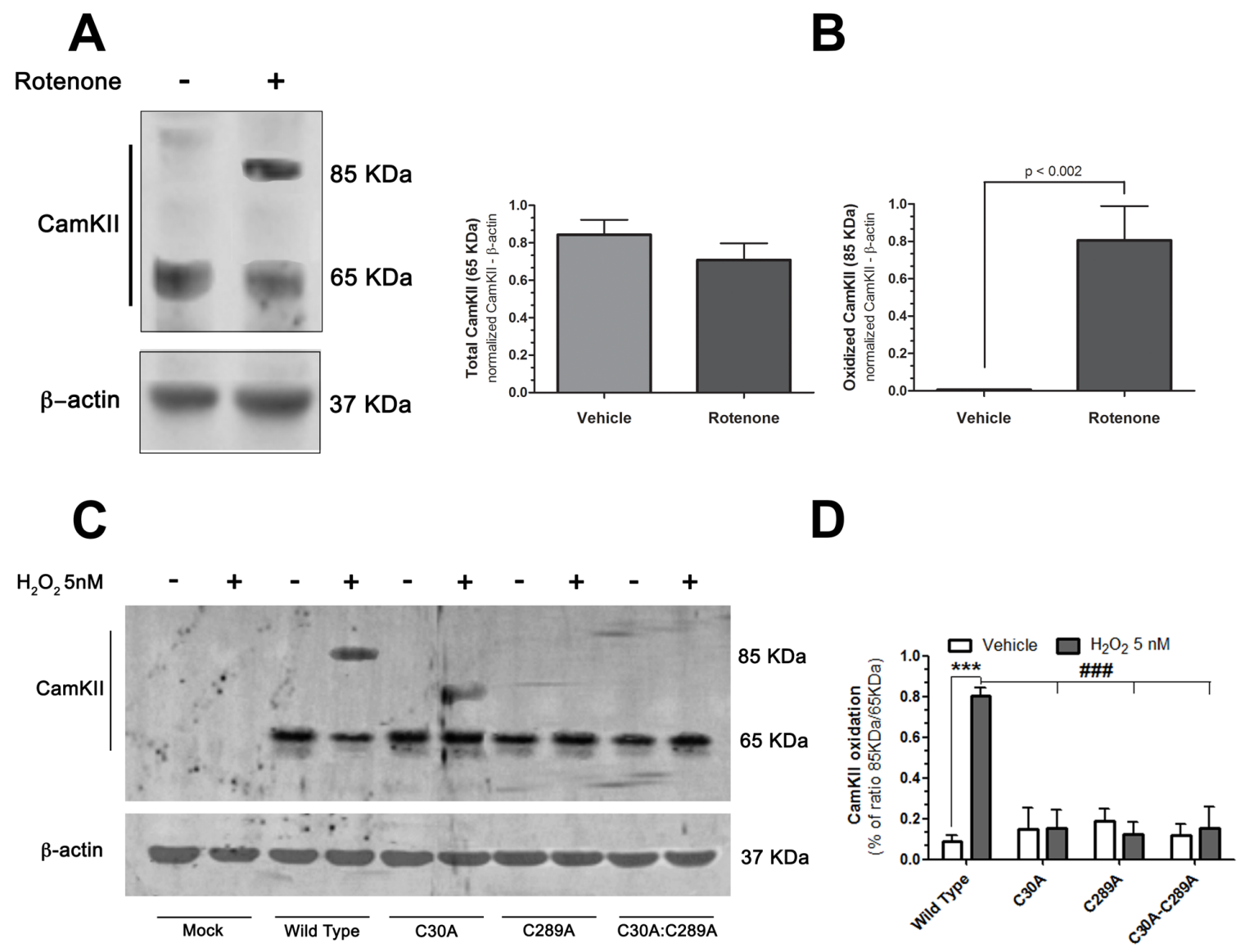

Figure 3

Oxidative stress-related disulfide formation in CaMKII A: Redox WB for CamKII revealed a 20KDa MW shift in lysates of ventral midbrain cultures exposed to $50 \mathrm{nM}$ rotenone for 24hours, suggesting the formation of a disulfide bridge. B: Quantification of the intensity of the bands shows no significative changes in CamKII expression and a significant increase of CaMKII in oxidized state (85KDa band) in $\mathrm{H}_{2} \mathrm{O}_{2}$ treated cells (2tailed unpaired t-test with Welch's correction; $p<0.002 ; n=3)$. C: Consistent with the results obtained in ventral midbrain cultures, redox WB of lysates from Drosophila cells expressing human wild type CamKIl $\alpha$ showed the formation of a disulfide bridge when cells were exposed to $5 \mathrm{nM}$ of $\mathrm{H}_{2} \mathrm{O}_{2}$ for 24 hours. The phenomenon was prevented in CamKII C30A and C289A mutants. Note that $\mathrm{H}_{2} \mathrm{O}_{2}$ treatment of cells expressing only the mutant $\mathrm{C} 30 \mathrm{~A}$ elicited the appearance of a $10 \mathrm{KDa}$ shift of CaMKIla, suggesting an oxidative process exerted only on C289. This may be explained by the computational evidence that C289 is more exposed to the solvent (see Figure 1, bottom panel: C289 is in the linker of the red subunit). Therefore, although C289 cannot form a S-S bridge with C30 in C30A mutant, its high exposure facilitates other kinds of thiol oxidation, such as S-NO formation. D: Quantification of band intensity expressed as ratio $85 / 65 \mathrm{KDa}$ (ONE-WAY ANOVA + Bonferroni test; $p<0.0001 ; n=3$ ) 
A

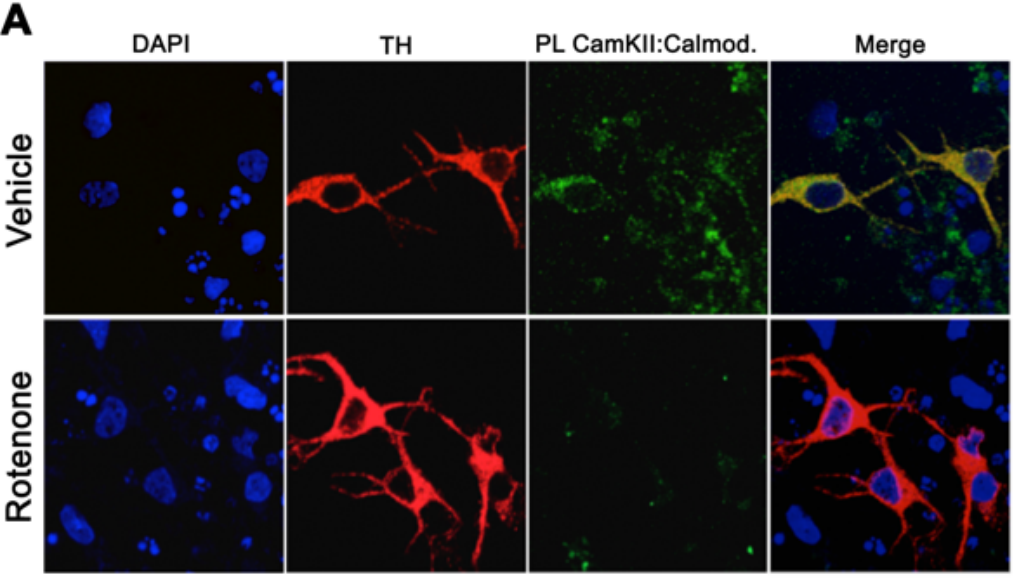

B
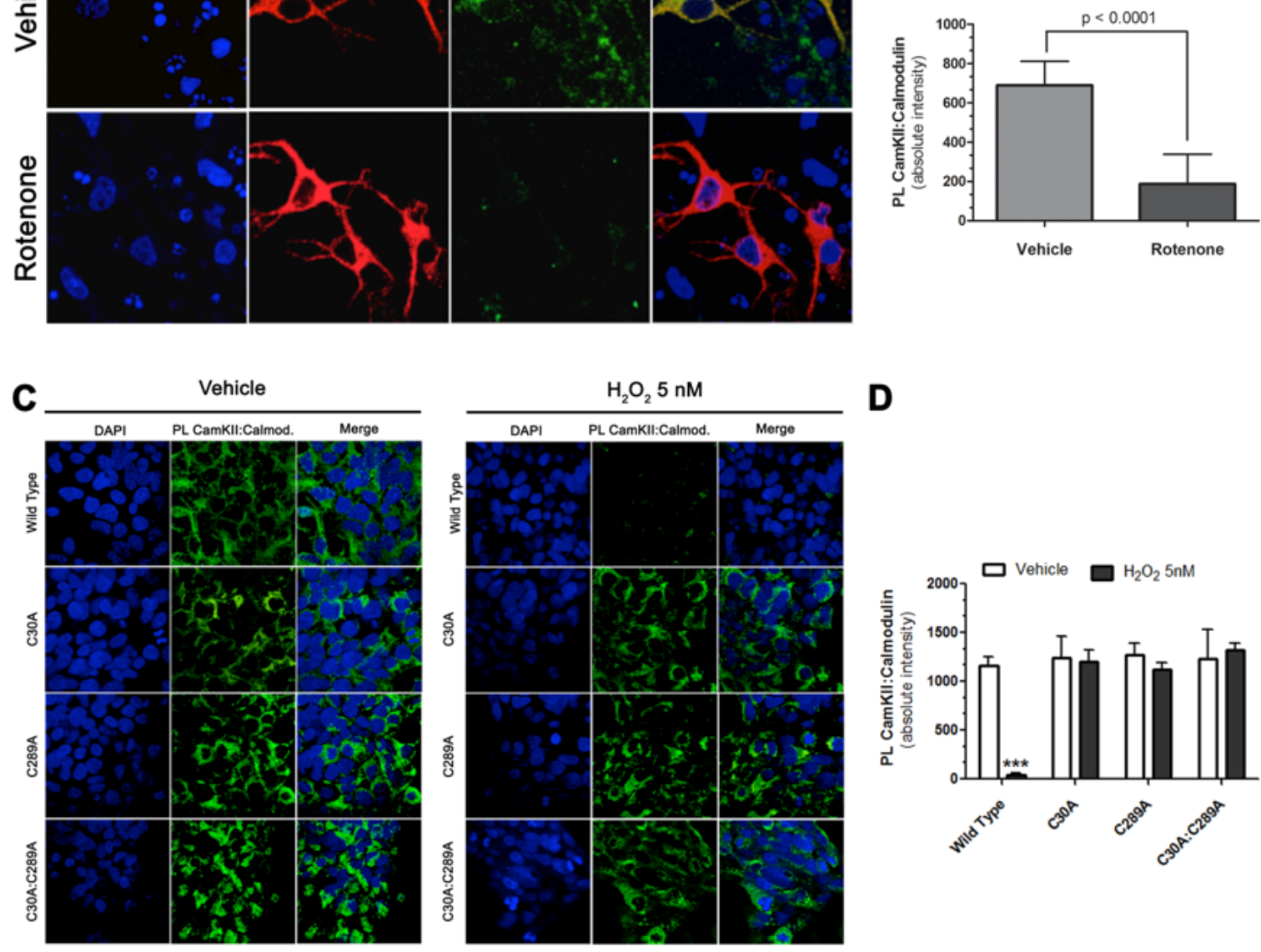

D

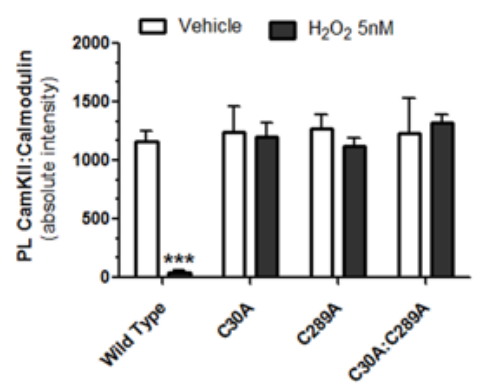

\section{Figure 4}

Disulfide formation between C\#) and C289 prevents CaMKII/CaM interaction A: Proximity ligation between CamKII and calmodulin in DA neurons of ventral midbrain primary cultures. In vehicle-treated cells, there is intense punctate signal for CamKII and calmodulin interaction. In contrast, rotenone treatment induced loss of fluorescence $\mathrm{PL}$ signal for $\mathrm{CaMKII-Ca}{ }^{2+} / \mathrm{CaM} \mathrm{B}$ : The plot reports the mean cellular PL fluorescence values (vehicle- or rotenone-treated). In each sample, PL signal was measured in 20-40 TH-positive neurons. Statistical testing by 2-tailed unpaired t-test with Welch's correction. C: Drosophila cells expressing human CamKII wild type, C30A, C289A or the double mutation C30A-C289A were exposed to $5 \mathrm{nM} \mathrm{H}_{2} \mathrm{O}_{2}$ for 24 hours. PL assay for $\mathrm{CaMKII}_{-} \mathrm{Ca}^{2+} / \mathrm{CaM}$ revealed, as in VMB cultures, the loss of signal for CaMKII$\mathrm{Ca}^{2+} / \mathrm{CaM}$ interaction. Noteworthy, all the mutations were able to prevent $\mathrm{H}_{2} \mathrm{O}_{2}$ mediated $\mathrm{CaMKII-Ca}{ }^{2+} / \mathrm{CaM}$ disruption, suggesting the critical role of the redox status of C30 and C289 in modulating the functional binding of $\mathrm{Ca}^{2+} / \mathrm{CaM}$. D: Quantification of

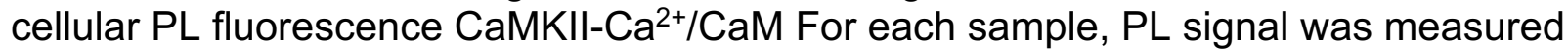
in 50-70 cells and the data are from 3 independent experiments. Statistical testing by ONE-WAY ANOVA with Bonferroni post-hoc test. 
A

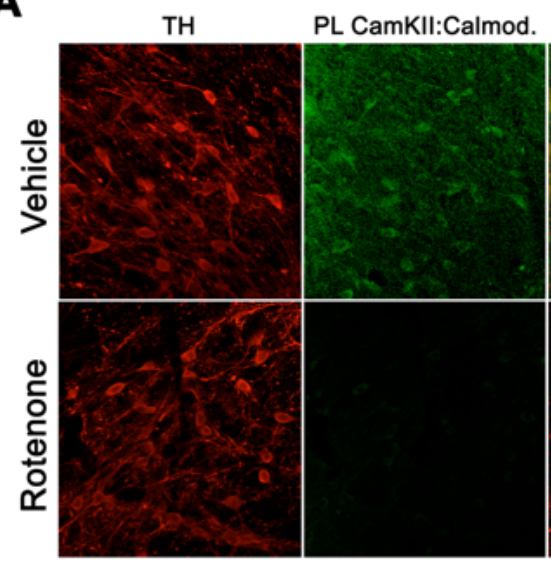

B

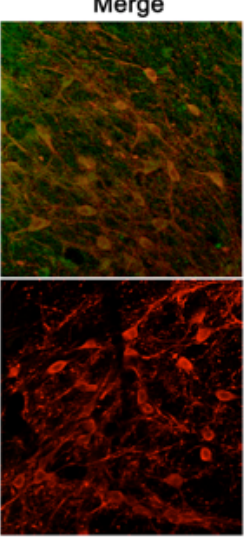

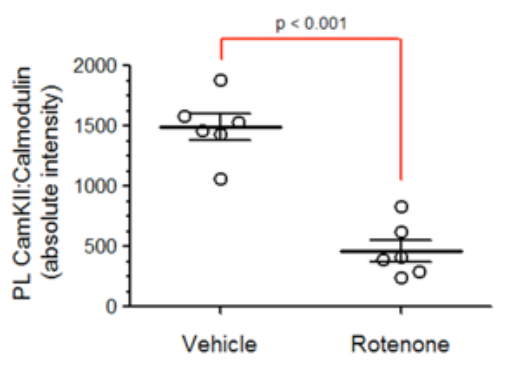

\section{Figure 5}

Evidence of disrupted CaMKII/CaM interaction in PD model of rotenone

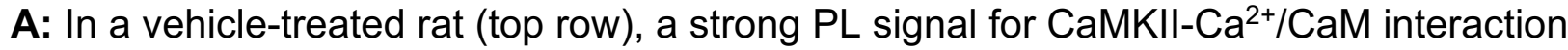
was detected. In contrast, rotenone treatment (bottom row), prevented PL signal CaMKII- $\mathrm{Ca}^{2+} / \mathrm{CaM}$, suggesting, again that, under mitochondrial injury and oxidative stress conditions, the two proteins lose the ability to interact. B: Quantification of PL fluorescence values for individual animals (vehicle- or rotenone-treated). Each circle represents the mean of PL fluorescence for a single animal. In each animal, PL signal was measured in 35-50 nigrostriatal neurons per hemisphere. Statistical testing by 2tailed unpaired t-test with Welch's correction. 


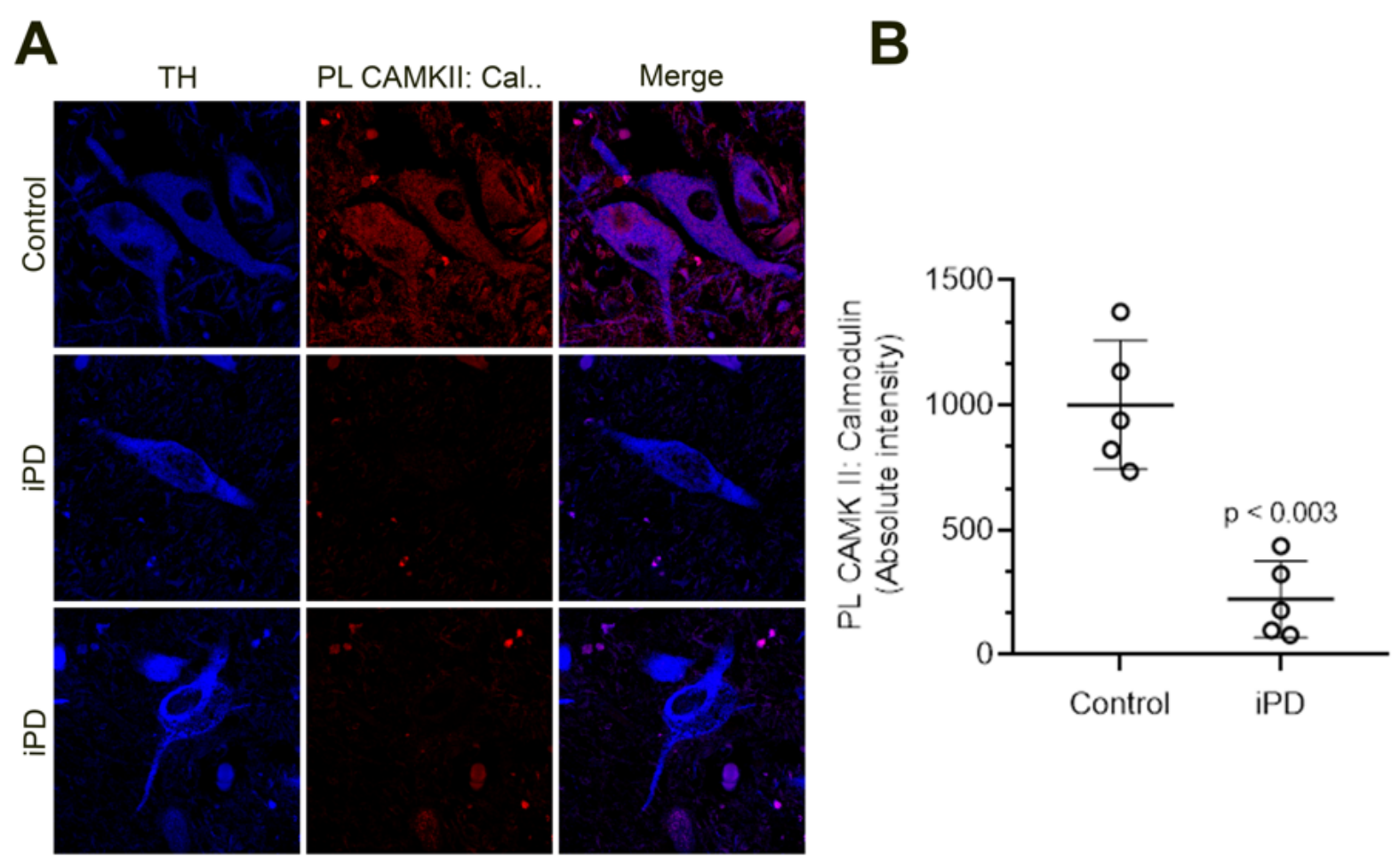

Figure 6

Relevance of CaMKII/CaM interaction in idiopathic PD

Proximity ligation between CamKII and calmodulin in human brain. A: In control patients (top row), a strong $\mathrm{PL}$ signal for $\mathrm{CaMKII-} \mathrm{Ca}^{2+} / \mathrm{CaM}$ interaction was detected. In contrast, idiopathic Parkinson's disease (iPD, bottom row), showed little PL signal CaMKII$\mathrm{Ca}^{2+} / \mathrm{CaM}$, suggesting, again that, under mitochondrial injury and oxidative stress conditions, the two proteins lose the ability to interact. B: Quantification of PL fluorescence values for individual patients (control or iPD). Each circle represents the mean of PL fluorescence for a single patient. In each patient, PL signal was measured in $35-50$ nigrostriatal neurons. Statistical testing by 2-tailed unpaired t-test with Welch's correction. 